\title{
Wnt-1-a key player in the regulation of human bone mass?
}

$\mathrm{E}$ arly-onset osteoporosis and osteogenesis imperfecta are associated with mutations in WNT1, new data reveal.

A number of loci associated with fracture risk have been identified in genome-wide association studies; however, the identified loci only modestly affect fracture risk. Consequently, the search for the 'missing heritability' of osteoporosis and other bone diseases continues.

Brendan Lee of the Baylor College of Medicine and the Howard Hughes Medical Institute and his team have pursued the identification of gene defects in the rare Mendelian disorder osteogenesis imperfecta to identify genes important in the regulation of bone mass. "The underlying hypothesis is that these genes may contribute to maintenance of postnatal bone mass," says Lee. "We have conducted a large whole-exome discovery project on families with osteogenesis imperfecta who are negative for previously described genes for this disorder."

The researchers report on clinical and genetic findings in one family with severe, early-onset and dominantly inherited osteoporosis and another family

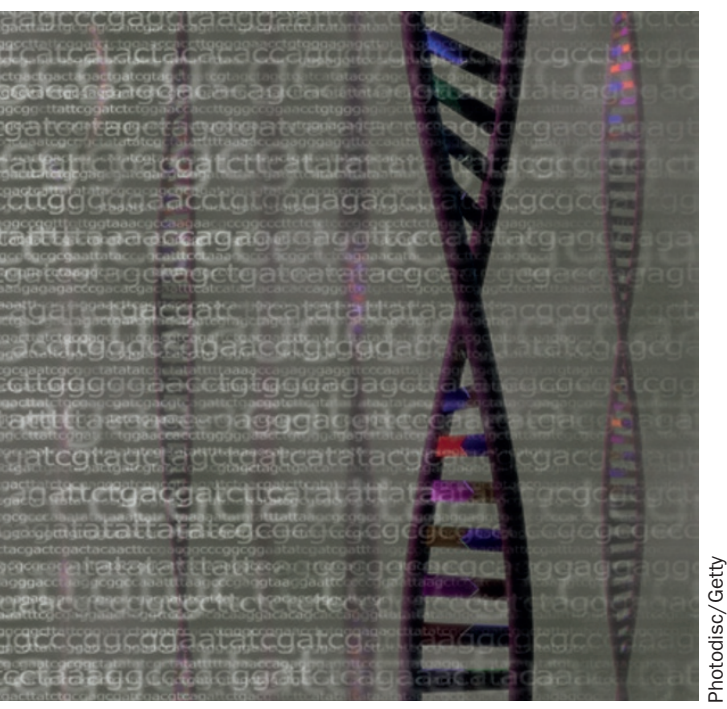

including two sisters affected by recessive osteogenesis imperfecta. The family with severe, early-onset osteoporosis includes 10 family members aged $12-68$ years with low BMD and vertebral or peripheral fractures. The other family includes one sibling with osteogenesis imperfecta and a first fracture at the age of 1 month who is now wheelchair-bound as a young woman owing to the severity of the bone disease. In her younger sister, prenatal, thirdtrimester, ultrasonography scans showed multiple fractures of the rib and femur; this individual, now a young woman, has severe intellectual disability with cerebellar malformation in addition to severe bone disease.

Genetic analyses identified mutations in WNT1 in affected members of both families. WNT1 encodes a signalling protein, Wnt-1, in the canonical Wnt pathway, which is an important signalling pathway in the regulation of bone formation and maintenance. In the family with early-onset osteoporosis, the researchers identified a heterozygous missense mutation within the conserved WNT motif of WNT1. In the family with osteogenesis imperfecta, both affected siblings were found to be homozygous for a nonsense mutation in WNT1.

"The dose-response is important," comments Lee. "Rarely do we have dose-response genetic changes for secreted signalling molecules. The semi-dominant inheritance of WNT1 mutations underscores this finding. When one mutation is present, you have early-onset osteoporosis; if two mutations are present, you have osteogenesis imperfecta." Interestingly, in the family with osteogenesis imperfecta, only one sibling had brain malformation; Wnt-1 has previously been implicated in brain development.

The researchers next investigated the effect of the WNT1 mutations in vitro and observed that both mutations reduce the capacity of Wnt-1 to induce canonical Wnt signalling. Furthermore, the mutant Wnt-1 proteins do not induce expression of downstream targets of Wnt signalling or stimulate mineralization to the same extent as wild-type Wnt-1.

The expression pattern of Wnt 1 in mice was then analysed to shed light on the role of the protein in regulating bone mass. Wnt1 mRNA expression was found in the brain, femur and spleen, as well as in bone marrow, in particular in cells of B-cell lineage and haematopoietic progenitor cells. In addition, lineage tracing experiments in transgenic mice showed expression of the gene in a subset of osteocytes. The researchers speculate that haematopoietic cells have a role in the regulation of bone formation and that Wnt-1 plays a part in the associated signalling pathway.

"Most researchers in the bone field would not have guessed Wnt-1 to be a key Wnt ligand controlling bone mass. In fact, its expression is relatively low in bone," comments Lee. "This observation suggests that key signalling proteins may not need to be expressed in great amounts but must be expressed at the right time and dose."

The team now plan to use mouse models to examine how Wnt-1 controls bone in a temporally and spatially restricted fashion. They are also investigating the possible use of Wnt-1 as a marker of bone formation in humans.

The findings could lead to the development of targeted therapies, for example, to alter Wnt signalling. As Lee highlights, "these data suggest that altered signalling is important in the pathogenesis of osteogenesis imperfecta, beyond matrix or collagen defects."

\section{Carol Wilson}

Original article Laine, C. M. et al. WNT1 mutations in earlyonset osteoporosis and osteogenesis imperfecta. N. Engl. J. Med. 368, 1809-1816 (2013) 